\title{
LA DIFERENCIA SEXUAL EN LAS NEUROCIENCIAS Y LA NEUROEDUCACIÓN
}

\author{
SONIA REVERTER-BAÑóN \\ Universitat Jaume I \\ reverter@uji.es \\ MARIA MEDINA-VICENT \\ Universitat Jaume I \\ medinam@uji.es
}

RESUMEN: Los argumentos neurocientíficos focalizados en demostrar diferencias sexuales en el cerebro gozan de una gran popularidad. Algunas de las conclusiones de dichos experimentos han derivado en propuestas neuroeducativas que promueven la educación segregada por sexos. Estas propuestas carecen del rigor necesario para poder ser aplicadas. No sólo porque los estudios neurocientíficos están lejos de poder aseverar diferencias sexuales significativas en el cerebro, sino porque falta un diálogo crítico entre las ciencias que fundamente estrategias educativas adecuadas en el ámbito de las diferencias sexuales del cerebro. Concluimos que es necesario establecer un diálogo entre los diferentes ámbitos que conforman la neuroeducación, porque solamente de este modo se pueden fundamentar modelos educativos democráticos donde la igualdad de género sea un valor irrenunciable.

PALABRAS CLAVE: género, sexo, educación, neuroplasticidad, discurso neurocientífico

SUMMARY: Neuro-scientific arguments focused on demonstrating sexual differences in the brain are very popular. Some of the conclusions of these experiments have derived in neuro-educational proposals aimed at fostering single-sex education projects. Such proposals are insufficiently rigorous for their application to be justified, not only because neuro-scientific studies are far from being able to identify significant sexual differences in the brain, but because the necessary critical dialogue between sciences that might sustain appropriate educational strategies is lacking. We conclude that there is a need to establish such a dialogue between the different areas involved in the neuro-education field. Only through a dialogue of this kind would we be able to base democratic educational models where gender equality is an inalienable value.

KEY WORDS: gender, sex, education, neuro-plasticity, neuro-scientific discourse

\section{Introducción: neurociencias y neuroeducación}

La filosofía de la educación se preocupa por analizar la educación humana y el modo ideal en el que debemos formarnos (especialmente en la infancia) para desarrollarnos plenamente como seres humanos 
y ciudadanos/as. Las neurociencias se definen como la "ciencia experimental, que con todas las herramientas técnicas disponibles y utilizando el método científico (observación, experimentación, hipótesis... ) tiende a explicar cómo funciona el cerebro, particularmente, el cerebro humano" (Mora 2007, p. 35). En la intersección de ambas disciplinas se encuentra el estudio de las variables neurocientíficas en los procesos de enseñanza-aprendizaje, cuyos hallazgos serán parte importante de la filosofía de la educación (Patten y Campbell 2011; Sousa 2011; Della Sala y Anderson 2012; Howard-Jones 2010, 2012; Busso 2013; Clark 2015; Joldersma 2016), al proponer teorías filosóficas para facilitar esos mismos procesos en su objetivo principal: la formación plena como seres humanos insertos en la sociedad.

Un ejemplo claro de esto se encuentra en Philosophical Reflections on Neuroscience and Education (2017), de William H. Kitchen, quien explora la relación entre educación y neurociencia y plantea la puesta en valor de los conceptos y planteamientos filosóficos a la hora de establecer este posible diálogo. Kitchen nos recuerda que la educación no se centra solamente en qué aprender sino en cómo aprender, y en esta línea, advierte que la neuroeducación se sostiene sobre una versión materialista modernizada de la filosofía mentalista del siglo XVII que resulta bastante problemática, ya que no hace más que reemplazar el concepto "mente" por el de "cerebro". A través de la filosofía de Wittgenstein, Kitchen argumenta en contra de la reciente preeminencia de la neurociencia en el discurso educativo y ofrece aclarar conceptos filosóficos básicos que a menudo quedan en el olvido o se malinterpretan en esta disciplina.

Con base en esta explicación, resulta necesario preguntarse si existe una relación jerárquica entre las neurociencias y las teorías filosóficas sobre educación (Bickle 2003), o si se trata de una influencia bidireccional (Gazzaniga 2012). Nuestra propuesta, siguiendo la idea de una relación dialógica entre disciplinas (Northoff 2004), es que la relación entre teorías filosóficas e hipótesis científicas es interdisciplinar (Castelli 2018), en el sentido de que implican algo más que la mera síntesis y adición de hipótesis. Es en esta relación simétrica e interdisciplinar entre filosofía de la educación y neurociencias (Bruer 2008) donde encontramos un nuevo término: neuroeducación. Si bien es cierto que los intentos de establecer puentes entre la investigación del cerebro y la educación son previos al escenario actual (Theodoridou y Triarhou 2009) y datan de finales del siglo XIX (Donaldson 1895; Post Halleck 1896), es en el siglo XXI, y particularmente des- 
pués de la denominada "década del cerebro" (1990-2000), cuando se presentan proyectos de aplicación neurocientífica en escuelas. ${ }^{1}$

A pesar de lo novedoso y del carácter a veces vago de los prefijos "neuro" (Vidal 2008), podemos definir la neuroeducación a partir de una concepción transdisciplinar como un campo de encuentro de miradas a la mente, al cerebro y a la educación, que plantea un diálogo entre teorías y metodologías (Ansari et al. 2011, 2012). Antonio M. Battro y Daniel P. Cardinali (1996) también establecen una definición de la neuroeducación que pone énfasis en su capacidad de entrar en contacto con otras metodologías y disciplinas: "una nueva interdisciplina y transdisciplina que promueve una mayor integración de las ciencias de la educación con aquellas que se ocupan del desarrollo neurocognitivo de la persona humana" (p. 1). Además de interdisciplinar, los autores consideran que la neuroeducación es transdisciplinar, que establece una nueva forma de integrar diferentes disciplinas que generen nuevas categorías conceptuales y prácticas. De este modo, queda claro que a pesar de que la neuroeducación bebe en gran parte de la neurociencia cognitiva, no se limita a ella (PallarésDomínguez 2013; Pallarés-Domínguez y Richart 2018), idea central si aspiramos a evitar lecturas reduccionistas de la neuroeducación.

Así pues, el objetivo principal de este encuentro entre neurociencias y educación es investigar las bases científicas y pedagógicas del proceso de enseñanza-aprendizaje (Nouri y Mehrmohammadi 2012) para aprovechar los conocimientos de las disciplinas convocadas al encuentro. Así que nuestra idea no es que la neuroeducación tenga que trasladar de forma automática las evidencias neurocientíficas al ámbito educativo (Maney 2014; Crifaci et al. 2015), sino que se trata de construir una concepción que supere ciertas limitaciones en la relación de ambas disciplinas (Brozek 2011). Y es que entender la neuroeducación como una disciplina que está a la espera de revelaciones neurocientíficas estaría fundamentando una relación de supeditación de la educación a los hallazgos neurocientíficos (Greenfield 2002). $\mathrm{Al}$ contrario, consideramos que el diálogo entre ambas disciplinas se debe desarrollar simétricamente, para no perder la riqueza específica de cada disciplina, que hace que del diálogo cooperativo no podamos esperar más que beneficios (Cortina 2011, pp. 48-51).

En resumen, con "neuroeducación" nos referimos al conjunto de disciplinas integradas en el programa de investigación mente-cerebroeducación que trabajan en un diálogo transdisciplinar, o en la metá-

${ }^{1}$ La "década del cerebro" fue una iniciativa patrocinada por la Biblioteca del Congreso y el Instituto Nacional de Salud Mental de Estados Unidos. 
fora propuesta por John Bruer $(1997,2008)$, creando puentes y no buscando fundamentos. La neuroeducación debe hacerse preguntas éticas antes de intentar "traducir" los hallazgos científicos a instrucciones educativas (Hardiman et al. 2012; Maxwell y Racine 2012). En este sentido, si bien el impacto de las neurociencias en la educación podría ser sustancial (De Vos 2016; Pallarés-Domínguez 2015), hay que ser precavidos, pues, como algunos autores revelan (Ferrari 2011; Schrag 2011), es improbable que el potencial para activar la educación por medio de mecanismos neurales en el aprendizaje altere sustancialmente la forma en que se enseña en el aula. Al menos por ahora, el carácter reciente de esta disciplina vuelve complicado imaginar la figura de un/a neuroeducador/a que pueda llevar al aula las aportaciones de la neurociencia de forma adecuada (Gardner 2008; Carballo 2016). Así que resulta imprescindible ser conscientes de que los/as educadores/as deben conocer de forma crítica las implicaciones que se derivan de la aplicación de la neuroeducación a las aulas (Campos 2010).

La intención central del presente artículo reside en identificar los riesgos que conlleva, para la fundamentación moral de una sociedad democrática (Salles 2013), otorgar excesivo crédito a los argumentos neurocientíficos sobre la diferencia entre los cerebros de mujeres y hombres en el ámbito educativo (Ansari et al. 2012). Y es que la traslación de la teoría del dimorfismo sexual a las aulas puede derivar en la segregación por sexos, lo que podría provocar un retroceso en la formación de una ciudadanía igualitaria, crítica y activa. Lejos de promover esta visión cerrada de los procesos de aprendizaje, consideramos que "comprender lo humano es comprender su unidad en la diversidad, su diversidad en la unidad" (Morin 1999, p. 25), y, por lo tanto, sin desvirtuar las aportaciones de la neuroeducación (Pallarés-Domínguez 2015), existe la necesidad de superar una visión reduccionista de la misma para iniciar diálogos transdisciplinares (Howard-Jones y Fenton 2012).

\section{En busca de las diferencias sexuales en el cerebro}

En las últimas décadas de profusión de investigación del cerebro, ha habido un interés creciente en demostrar diferencias cerebrales marcadas por el sexo, tal y como lo muestran los más de 17 mil estudios sobre dicho tema publicados en PubMed. ${ }^{2}$ En la mayoría

\footnotetext{
${ }^{2}$ PubMed es un motor de búsqueda de libre acceso a la base de datos MedLine (con 4800 revistas científicas) de citaciones y resúmenes de artículos de investigación biomédica, ofrecido por la Biblioteca Nacional de Medicina de Estados Unidos.
} 
de los casos, estas supuestas diferencias cerebrales entre los sexos no solamente se usan para fundamentar una distinción esencial entre mujeres y hombres, sino también entre personas heterosexuales y homosexuales, así como entre personas cisgénero y transgénero. Aun así, los estudios no son concluyentes respecto de si el sexo (y sus diferencias) se refleja de manera consistente en el cerebro, tanto en su estructura como en su función (Grabowska 2017), con lo que dicha avalancha de datos acaba por permitir interpretaciones ambiguas o contradictorias (Hyde 2005; Tan et al. 2016).

En medio de esa ambigüedad encontramos dos posturas contrapuestas: aquella que rechaza la posibilidad de hablar de diferenciación sexual del cerebro de forma indudable, consistente y probada; y aquella que arguye que el cerebro presenta signos claros de dimorfismo sexual. Como la ciencia no puede avanzar sin resultados corroborados de forma indudable, habrá que ser muy cautos a la hora de realizar interpretaciones concluyentes sobre si los experimentos son replicables o no. Consideramos que al día de hoy, y aunque han sido probadas ciertas diferencias sexuales en el cerebro, éstas no son ni concluyentes ni consistentes, ${ }^{3}$ y mucho menos son diferencias que puedan explicar patrones comportamentales de forma contundente y científicamente avalada; es decir, que las diferencias encontradas están lejos de poder ser correlacionadas con aspectos mentales, actitudinales o de comportamiento diferenciado entre sexos.

Veamos dos ejemplos de publicaciones científicas que han abordado este tema en números monográficos. La revista Journal of Neuroscience Research publicó (enero de 2017) un número pionero dedicado exclusivamente al tema de la diferencia sexual en el cerebro con el prometedor título de An Issue Whose Time Has Come: Sex/Gender Influences on Nervous System Function. Aunque se parte

${ }^{3}$ Conviene recordar que en 2016 se publicó un estudio en la prestigiosa revista PNAS (Proceedings of the National Academy of Sciences of United States) en la que sus autores, Eklund, Nichols y Knutsson (2016), alertaban de fallos en las imágenes de cerebros por fMRI. Un fallo en el software, unido al escaso rigor en la práctica científica, podría haber contaminado miles de imágenes que iluminaron por error zonas del cerebro aun cuando no hubiera activación. Esto podría haber dado pie, en las últimas dos décadas, según los autores, a miles de estudios y conclusiones totalmente equivocados, entre los que se estima un $70 \%$ de falsos positivos. Como advierten: "Estos resultados cuestionan la validez de una serie de estudios de fMRI y podrían tener un gran impacto en la interpretación de resultados de neuroimagen débilmente significativos" (Eklund et al. 2016, p. 7900; la traducción es nuestra). A esto se ha de sumar la llamada "crisis de la reproducibilidad", que según un estudio publicado en Nature (Baker 2016) supone que el $70 \%$ de los científicos han fallado a la hora de reproducir experimentos de otros. 
de la voluntad de afirmar tales diferencias, como se explicita en el editorial de la revista (Prager 2017) y en el del monográfico (Cahill 2017), mostrando principalmente experimentos que corroboran una diferencia sexual en el cerebro, la gran mayoría de los 70 artículos que contiene el número monográfico reconoce los límites a la hora de aseverar resultados concluyentes respecto de cómo interpretar tales diferencias en el cerebro.

Asimismo, con el revelador título de From Sex Differences in Neuroscience to a Neuroscience of Sex Differences: New Directions and Perspectives, la plataforma de revistas y publicaciones científicas Frontiers in Neuroscience publicó un número monográfico (septiembre de 2015) sobre diferencias sexuales en el cerebro con base en los circuitos hormonales. Tal y como Pletzer $(2015$, p. 6) explica en el editorial, las inconsistencias que impiden aseverar y concluir diferencias sexuales en el cerebro de forma contundente se asocian a dos problemas: el reducido tamaño de las muestras, y la gran variación en las metodologías utilizadas en los experimentos.

$\mathrm{Al}$ consultar estos dos monográficos en revistas científicas de indudable trayectoria, nos damos cuenta tanto del creciente interés de las neurociencias en las diferencias sexuales del cerebro, como de las dificultades para interpretar las diferencias encontradas. Veamos cuáles son éstas con el objetivo de poder calibrar la oportunidad de una educación diferenciada por sexos.

\section{Los argumentos neurocientíficos sobre la diferencia sexual}

Con la cautela necesaria a la hora de abordar los resultados neurocientíficos sobre las diferencias sexuales de los cerebros, debemos advertir una práctica que parece producirse de manera generalizada en los últimos años: el intento de correlacionar esas diferencias con necesidades educativas diferentes. Como acabamos de comentar, la mayoría de los estudios no logran corroborar una diferencia sexual en el cerebro tan consistente que pueda explicar patrones cognitivos diferenciados. Por ello, parece tratarse de una hipótesis no demostrada (Eliot 2013), mantenida en gran parte por el imaginario social (Vidal 2005, 2011), que influye en lo que pensamos acerca de las necesidades educativas de niñas y niños. Veamos algunas de estas creencias, con el objetivo de ilustrar la espinosa cuestión a la que nos estamos refiriendo. ${ }^{4}$

\footnotetext{
${ }^{4}$ Muchos de los resultados de diferentes experimentos neurocientíficos se han lanzado a la opinión publica de forma acrítica y se han convertido en parte de la cultura popular; sin embargo, cabe señalar que, en muchos casos, tales resultados pueden
} 
En primer lugar, nos ocuparemos del argumento sobre el tamaño del cuerpo calloso (tejidos que unen los dos hemisferios); según una creencia basada en la ciencia popular, es más voluminoso en las chicas adolescentes que en los chicos del mismo rango de edad. Algunos científicos como Michael Gurian y Kathie Stevens (2011) sostienen que el cerebro femenino, al contener un mayor tejido de cuerpo calloso que permite la interconectividad entre hemisferios, contaría con una mayor capacidad multitarea que el cerebro masculino. En relación con esto tuvo mucha repercusión la investigación de Ingalhalikar y otros (2014) acerca de la estructura de las conexiones del cerebro humano. Las conclusiones, basadas en la técnica Diffusion Tensor Imaging (DTI) aplicadas a 949 jóvenes, establecían que los cerebros masculinos son mejores en la comunicación intrahemisférica, y los femeninos en la comunicación interhemisférica:

Las observaciones sugieren que los cerebros masculinos están estructurados para facilitar la conectividad entre percepción y acción coordinada, mientras que los cerebros femeninos están diseñados para facilitar la comunicación entre modos de procesamiento analíticos e intuitivos. (Ingalhalikar et al. 2014, p. 823; la traducción es nuestra.)

Ese estudio fue muy criticado (Joel y Tarrasch 2014; Hanggï et al. 2014) y se intentó replicar utilizando una nueva técnica, el Resting State fMRI (Satterthwaite et al. 2014). A pesar de las miles de conexiones que el estudio puso de manifiesto, sólo un 0.51 por ciento de ellas mostraban una diferencia entre los sexos, es decir, una cantidad minúscula. Los autores de este último estudio admiten, al final de su trabajo: "Si bien existen diferencias de sexo en la conectividad, los modelos de conectividad de los cerebros masculinos y femeninos son, en general, más similares que diferentes" (Satterthwaite et al. 2014, p. 2392; la traducción es nuestra). La comunidad científica sigue manteniendo por ello, que el tamaño del cuerpo calloso está en relación con el tamaño del cerebro completo y no tanto con el sexo (Eliot 2013; Leonard et al. 2008; Luders y Toga 2010). Pese a estas advertencias, se sigue pensando acríticamente que esas minúsculas diferencias son suficientes para hablar de diferencias sexuales significativas en relación con el cerebro, su funcionamiento, y su posible impacto en el proceso de aprendizaje entre mujeres y hombres.

encontrarse en entredicho debido a la insuficiencia de evidencias y la existencia de prejuicios en el establecimiento de conclusiones (Reverter-Bañón 2016, pp. 966-969; 2017b). 
En segundo lugar, nos encontramos con el argumento de la lateralización cerebral. Con respecto a esta cuestión, existe la creencia de que la función neural de los niños es más lateralizada; es decir, que los niños usan el lado derecho o el izquierdo del cerebro, uno a la vez, mientras que las niñas utilizan los dos hemisferios al mismo tiempo. Dicha creencia, extensamente divulgada, está basada en un estudio realizado con 19 chicas y 19 chicos (Shaywitz et al. 1995), en el que se les asignaban tres diferentes tareas lingüísticas; en una de ellas, los 19 chicos activaron sólo el área del lóbulo inferior frontal izquierdo, mientras que las 19 chicas activaron ambos lóbulos frontales. Este experimento tuvo mucha repercusión en los medios de comunicación de masas (Bing 1999; Ayala et al. 2014). Sin embargo, desde entonces (1995) se han realizado múltiples experimentos en la misma línea (con un total de 1526 sujetos estudiados) y en todos los casos la conclusión es que el proceso de lateralización es mucho más complejo de lo que las diferencias entre sexos indican (Sommert et al. 2008). Es decir, dicho fenómeno se debe explicar teniendo en cuenta otros factores que influyen en que el individuo, masculino o femenino, desarrolle un tipo de lateralización u otra.

En tercer lugar, y con respecto al sistema nervioso autónomo y el hipotálamo, se arguye que hay en este último un dimorfismo sexual en la región INAH-3, algunas diferencias en los niveles neuroquímicos y en la expresión de los receptores hormonales (García-Falgueras y Swaab 2008). Sin embargo, y aceptando las diferencias sexuales atribuidas a las hormonas (Swaab y García-Falgueras 2009), hay que ser cautos a la hora de sacar conclusiones; pues no se ha podido probar de forma definitiva que estas diferencias tengan relación con diferencias comportamentales concretas (Eliot 2011). De hecho se desconoce, como el estudio de García-Falgueras y Swaab (2008) lo indica, qué parte de la identidad en toda su complejidad estaría representada en esa porción del hipotálamo, la región INAH-3. No obstante, la ciencia popular suele afirmar que la química hormonal comporta diferencias "enormes" entre los sexos (Schulz y Katime 2003), y esta tesis suele ser la más importante para los defensores de la educación segregada por sexos (Gurian y Stevens 2011).

Estas creencias generalizadas suelen acompañarse de una lista de errores de intención y de prejuicio en la práctica neurocientífica. En este sentido, suele producirse una exageración de datos, ya que a veces, partiendo de experimentos con pocos sujetos, se llega a conclusiones universales, como lo ha denunciado la misma comunidad científica (McCabe y Castel 2008; Gonon et al. 2001; Button et al. 2013). Como ejemplo podemos citar el caso de la neuróloga Louann 
Brizendine (2006), quien concluyó que las mujeres pronuncian 20 mil palabras por día, mientras que los hombres sólo 7 mil, habiendo realizado un experimento con 5 niñas y 5 niños.

Además, otro de los errores se refiere a que con base en diferencias físicas mínimas y no corroboradas se extrapolan conductas psicológicas cuyo nexo con lo biológico no está fundamentado (Jordan-Young 2011). Sin embargo, si nos remitimos a estudios con muestras más grandes y con una mayor cautela en la extrapolación de sus resultados, vemos que las aseveraciones formuladas de manera concluyente sobre las diferencias cerebrales de los sexos pueden ponerse en duda en muchas de sus dimensiones (Bell y Variend 1985; Bishop y Wahlsten 1997; Bluhm 2012; Kraus 2012; Pletzer 2015; Marwha et al. 2017). De lo dicho podemos extrapolar ciertas cuestiones que nos incitan a poner bajo la lupa las aseveraciones tajantes que a veces se emiten desde el terreno de las neurociencias, y que planteamos a continuación:

En primer lugar, se ha podido comprobar la existencia de una tendencia a la exageración de resultados, con una universalización incongruente y acientífica (Gonon et al. 2001; Eliot 2011, p. 897; McCabe y Castel 2008; Joel y Tarrasch 2014).

En segundo lugar, en la mayor parte de los casos, los experimentos se realizan con pocos sujetos y no son corroborados (Button et al. 2013).

En tercer lugar, existe el peligro de usar expectativas para crear la diferencia sexual (e indirectamente la de género) que la ciencia no demuestra (Fine 2008; Vidal 2011; Bian et al. 2017).

En cuarto lugar, la imposibilidad de trasladar resultados de experimentos con adultos a infantes; algo que podemos observar en los experimentos de estudios funcionales MRI. En los pocos casos en que éstos se han querido replicar en niños/as no se ha llegado a los mismos resultados, e incluso a veces los resultados han mostrado que la diferencia entre infantes y adultos es más grande que la que puede haber entre sexos (Fine 2010; Eliot 2011, 2013; Kaiser 2012).

En quinto lugar, se puede producir la creación de neuromitos (Geake 2008), interpretaciones erróneas de datos científicos acerca de cómo funciona el cerebro (Mora 2007, 2013; Howard-Jones 2014).

Por último, en algunos casos las extrapolaciones de lo físico y biológico (sexo) a lo comportamental (género) pueden conllevar actitudes que nos hacen bajar la guardia con respecto a las desigualdades de género, tal y como señala Fine: "La respuesta, 'Oh, es el cerebro', ofrece una justificación ordenada para aceptar el statu quo teniendo la conciencia tranquila" (2008, p. 71; la traducción es nuestra). 
En resumen, las lecturas reduccionistas de algunos experimentos en el ámbito de las neurociencias suponen un riesgo en lo que atañe a proyectar modelos educativos eficientes para una sociedad igualitaria (García-Marzá y Feenstra 2013).

\section{Las categorías de sexo y género}

En el debate sobre la conveniencia o no de la educación diferenciada por sexos con base en los descubrimientos de las neurociencias debemos, como corolario, dar un pequeño repaso a los conceptos de sexo y género. Se considera al género (en binomio con el sexo) como el principal elemento de continuidad de los roles sociales, y el principal factor de categorización social de niñas y niños (Caplan y Caplan 1994; Carothers y Reis 2013). A diferencia de un concepto estable, el género se encuentra en continua discusión dentro de las corrientes feministas (Scott 1986; Tubert 2011; Fraisse 2016). Dado este debate, ignorado generalmente en las neurociencias, la visión que se tiene y el uso que se hace del concepto "género" en estas ciencias pueden resultar reduccionistas o inadecuados (Jordan-Young y Rumiati 2011; Rippon et al. 2014).

Una de las prácticas habituales en el ámbito de las neurociencias (y también de la neuroeducación) es asimilar el concepto de género al de sexo (Dussauge y Kaiser 2012). De este modo, se analiza la división de género partiendo de la idea de que es la consecuencia de la dualidad de sexos. No existe, por ello, un acercamiento crítico que ponga en duda esa linealidad, ya que justamente para hablar de educación en y para la igualdad habría que "deshacer el género" (Butler 1990, 2004) como estructura de un patriarcado que construye la desigualdad a través de los patrones de género (Pateman 1988; Johnson 1997; Lindsey 2015). Así, es necesario explorar si existe una base científica que otorgue sentido a la separación de los sexos en la educación; y para ello será necesario revisar si existe una diferenciación sexual presocial en aquellos elementos que intervienen en el proceso enseñanza-aprendizaje en el aula.

Desde el debate feminista de las últimas décadas (Reverter-Bañón 2010) se intenta establecer en qué medida lo que se ha entendido como "sexo biológico" no es también "sexo construido", precisamente construido a la par que se construye el género (Butler 1990, 2004). Desde este punto de vista, la "normalidad de los dos sexos" no lo es por ser natural, sino por la convención de normas sociales que dicta que lo es (Medina-Vicent 2016). De hecho, a veces, como en los casos de intersexualidad, incluso el protocolo médico vela por una 
normalización que borre lo natural del cuerpo (Fausto-Sterling 2000, 2015), justificando intervenciones sobre los cuerpos de bebés intersexuales (Reverter-Bañón 2017a). De modo que podemos hablar de dos tecnologías diferenciadas, una genética esencialista y otra quirúrgica constructivista (Preciado 2002; Flor 2006; Balza 2009). Así, mientras que las posturas que están en contra de la segregación tienen una visión más constructivista del género, y lo entienden como algo que emerge en relación con el contexto social, y que acaba afectando al cerebro (el género como constructo social va dejando su huella en el cerebro), aquellas posturas que defienden la segregación mantienen una posición más esencialista sobre el género, entendiéndolo como una esencia inherente y diferenciada que tendría su base en el sexo de los sujetos (el sexo, como algo biológico presente en el cerebro, determina el género social).

Son, como vemos, dos interpretaciones diferentes sobre cómo entender la relación entre sexo biológico y género social. Teniendo en cuenta estas aportaciones de la teoría feminista, sería necesario revisar cómo se utilizan las categorías de sexo y género en los estudios neurocientíficos, que son la base interpretativa para las propuestas neuroeducativas, y muy especialmente para la propuesta de la segregación sexual en el aula. Así, las categorías de sexo y género remiten inexorablemente a los conceptos más amplios de biología y cultura. En las propuestas neuroeducativas relacionadas con las diferencias sexuales será científicamente relevante mantener con claridad el uso de tales categorías, dejando atrás el baile conceptual que abunda en la bibliografía neurocientífica (Kaiser et al. 2009; Jordan-Young y Rumiati 2011; Joel 2016), donde a las diferencias comportamentales entre mujeres y hombres a veces se les llama "diferencias de sexo" y a veces "diferencias de género" (Kaiser 2012; Rippon et al. 2014). Esta indecisión, o confusión, revela el desacuerdo con respecto a si las diferencias comportamentales son atribuibles al sexo del cerebro, o si son explicables por las diferencias en la cultura de los géneros (Schmitz 2012).

Si bien una corriente esencialista ha provocado hablar de diferencias sexuales como una categoría puramente natural (Haslam et al. 2000; Haslam y Whelan 2008), sería necesario depurarlas de los elementos de género construidos por las culturas. Además, y aunque en un principio una argumentación puramente biológica de las diferencias sexuales supuestamente fundamentada en estudios empíricos no defendería una desigualdad sustancial en el papel social de mujeres y hombres, la interpretación esencialista que se ha derivado de estas lecturas reduccionistas sí puede acabar promoviendo roles desiguales 
(Vidal 2005, 2011; Hoffman y Bluhm 2016). Consecuentemente, el problema se generaría en el momento en que las supuestas diferencias encontradas en los cerebros de mujeres y hombres se acabaran interpretando en clave de desigualdad social, perpetuando modelos de género dicotómicos y esencialistas. Una cuestión por debatir en el ámbito científico es cómo pueden estar influyendo dichas premisas sexistas en los estudios sobre neuroeducación (Fine 2010).

Desde una perspectiva ética, la incorporación acrítica, por parte de las instituciones educativas, del argumento del dimorfismo sexual puede suponer un riesgo para el fomento de sociedades democráticas. Hasta ahora la neuroeducación, como nueva disciplina en formación, no se ha decantado de manera clara respecto del dimorfismo sexual. $\mathrm{Y}$ es que, como hemos dicho ya, no contamos con una afirmación científica clara que pueda establecer de forma concluyente tal dimorfismo. No hay, por lo tanto, consenso dentro de esta joven disciplina, y por ello se hace necesario el diálogo interdisciplinar que aquí proponemos.

En este diálogo entre neurociencias y educación será prioritario preguntarse por la fundamentación moral en las decisiones educativas, renunciando a cualquier pretensión reduccionista. En este sentido, si bien es cierto que las diferencias psicológicas y psicosociales entre los géneros pueden llegar a ser grandes y están bastante bien descritas, las diferencias en el cerebro no son tan evidentes. Estudios recientes (Joel et al. 2015; Bian et al. 2017) sostienen que tales diferencias son pequeñas y están muy influidas por la cultura y la educación y, sin embargo, se continúa con la tendencia a mostrar un mapa binario del cerebro humano, aun cuando los datos revelan que hay más similitudes que diferencias. Tampoco resulta obvia ni científicamente probada la correlación entre las diferencias psicosociales (género) y las diferencias cerebrales (sexo) (Jordan-Young 2011; Fine 2010; Hyde 2007). A estas inexactitudes se ha de sumar, como ya hemos comentado, la tendencia de la literatura no científica de divulgación a interpretar de manera exagerada y distorsionada los hallazgos neurocientíficos sobre las diferencias cerebrales entre mujeres y hombres (Eliot 2011; Johnson 2011; Bluhm 2012). ${ }^{5}$

Por todo ello y a la luz de los avances de las neurociencias críticas a este respecto, nuestra propuesta es que los conceptos sexo y género

${ }^{5}$ Un ejemplo de esta divulgación acrítica de los resultados neurocientíficos sobre las diferencias cerebrales de los sexos entre el público general se encuentra en la literatura popular del management dirigida a mujeres, que se nutre de dichos argumentos para sostener una forma diferente de liderazgo de las mujeres basada en sus estructuras cerebrales (Medina-Vicent y Pallarés-Domínguez 2017). 
funcionan en relación (Haraway 1990; Fausto-Sterling 2000; Dalton y Ortegren 2011; Kaiser 2012; Schmitz y Höppner 2014; Hoffman y Bluhm 2016), y que la mejor opción será referirnos a ellos con la fórmula sexo/género. Con dicha fórmula dejamos abiertas dos cuestiones que siguen sin estar científicamente determinadas: (a) si podemos seguir hablando de un modelo enteramente dimórfico del cerebro, como la neuropsicóloga Joel (2015) y su equipo recientemente se han preguntado, al hablar de "cerebro intersexo" (como lo indica el título de su artículo), o continuum o "mosaico", 6 como el modelo más adecuado para hablar del cerebro humano; y (b) si podemos seguir manteniendo la dicotomía naturaleza-cultura, como van Anders y Dunn (2009) se preguntan al estudiar la influencia de las hormonas; o como Halpern y LaMay (2000) ponen en duda al demostrar que variables medioambientales alteran los fundamentos biológicos de la inteligencia.

\section{Conclusión: la neuroplasticidad y su potencial crítico}

Lo dicho nos lleva, por lógica, a la tesis de la neuroplasticidad (James 1890), entendida como la capacidad del cerebro humano de organizarse, remodelarse y cambiar. Dicha plasticidad se puede manifestar tanto en el nivel de cambios en las neuronas, o de todo el cerebro, tal como ocurre en el remapeo cortical como respuesta a daños cerebrales (Pascual-Leone et al. 2005, 2011). Lo importante de esta teoría, en lo que se refiere al desarrollo de nuestro trabajo, es la aportación fundamental de que el cerebro humano no está enteramente "cableado" (hardwired) con circuitos neuronales fijos, sino que está sujeto a cambios constantes. Esto supone que las estructuras y funciones del cerebro humano no son una realidad acabada. Por ello, en el caso de que realmente existiesen diferencias considerables entre los cerebros de mujeres y hombres, éstas no tendrían por qué estar condicionadas de forma natural ni definitiva.

Al contrario, esta tesis afirma que hay muchos ejemplos de recableado cortical y subcortical en los circuitos neuronales en respuesta al entrenamiento o a daños cerebrales. La neurogénesis, o nacimiento de células cerebrales, se acepta cada vez más como una evidencia que ocurre en los cerebros de mamíferos a lo largo de la vida (Rakic 2002). Experimentos recientes revelan que no sólo el hipocampo y el bulbo olfativo son susceptibles de cambios, sino que otras partes del cerebro, incluido el cerebelo, muestran capacidad neurogenerativa

\footnotetext{
${ }^{6}$ Véase la propuesta del cerebro mosaico de Garcia-Falgueras et al. 2005.
} 
(Ponti et al. 2008). Algunos ejemplos de la neuroplasticidad se encuentran en los trabajos de Eleanor Maguire (2000) con los taxistas de Londres, quienes aumentaban su hipocampo al tener que memorizar el complejo mapa de dicha ciudad. En esta misma línea estarían los experimentos de Thomas Elbert y otros (1995), y de Álvaro PascualLeone y otros $(2005,2011)$. Todos ellos son considerados relevantes para señalar cómo esa neuroplasticidad no sólo se activa con la práctica, como en los dos experimentos señalados, sino también con la imaginación y las expectativas (Davidson y Begley 2012).

Al ser la organización del sistema nervioso humano, tanto en su sentido funcional como organizacional, un proceso continuo y dinámico, entendemos que nuestra experiencia depende de esa plasticidad (Evers 2010; Wexler 2011; Fine et al. 2013; Lipina 2014). La cuestión será organizar un sistema educativo capaz de afectar esa plasticidad y aprovecharse de ella en línea con los valores que de forma consensuada elijamos como los humanamente mejores (Tokuhama-Espinosa 2011). ${ }^{7}$ En este sentido, y aceptando que pueden existir diferencias cerebrales entre sexos (o entre otras categorías biológicas), la neuroeducación habrá de decidir qué diferencias quiere tomar en cuenta, cuáles son lo suficientemente consistentes para sugerir estrategias educativas diferenciadas, y cuáles conviene integrar en un sistema educativo cuyo objetivo sea la igualdad entre individuos, independientemente de su sexo y género.

Frente a las derivaciones reduccionistas que intervienen en los estudios neurocientíficos en la educación, es más necesario, si deseamos defender una educación ética e igualitaria, "investigar qué es y cómo funciona la neuroeducación, y cómo esta disciplina puede ayudar y contribuir a la educación en virtudes" (Codina 2014, p. 169). Y es que difícilmente podrá conseguirse una educación igualitaria si aceptamos los presupuestos neurocientíficos de forma acrítica. Además, si educamos a niños y niñas de manera diferenciada, supuestamente según las capacidades que con más facilidad puedan desarrollar en razón de sus estructuras cerebrales, correremos el riesgo de acabar reproduciendo viejos modelos dicotómicos de género, de mujeres y hombres.

Nuestro objetivo no es simplemente descartar el debate sobre la neuroeducación en función de su incapacidad actual para convencer-

${ }^{7}$ Esta tesis nos remite al concepto de "epigénesis proactiva" de Kathinka Evers (2015), quien lo define como una forma de dirigir la evolución para que influya en las improntas culturales que se almacenan en nuestro cerebro. Véase PallarésDomínguez (2016) para un análisis de este concepto en relación con la neuroeducación. 
nos de que los cerebros de niños y niñas pueden aprender más y mejor por separado que juntos. Por otra parte, nuestros argumentos no anulan el interés de un campo de estudio e investigación como es la neuroeducación, sino que lo promueven, entendiéndolo como una posibilidad que puede ser muy fructífera y relevante si logra conformarse como un ámbito de conocimiento que surge del diálogo interdisciplinar; que compone sus objetivos de forma transdisciplinar - es decir, creando a la vez una nueva disciplina - y si hace todo lo anterior sin perder de vista la principal aspiración de la educación. Defendemos que esta aspiración supone comprender de qué forma podemos educar para construir un mundo más respetuoso con la diversidad. Educar para un aprendizaje que haga florecer las mejores capacidades individuales que cada persona pueda tener es parte de ello, y dicha misión tiene mucho más que ver con las políticas educativas que cada Estado pueda emprender, que con las implicaciones neuronales de cada niño y cada niña. En todo caso, éstas han de poder ser estudiadas y comprendidas en una cartografía más amplia que la que nos pueden dar las neurociencias sobre los cerebros.

En conclusión, las decisiones que atañen a la educación involucran más que evidencia empírica (Liben 2015, 2016; Derks y Krabbendam 2013; Jensen 2010), en especial, debates y consensos sobre valores y propuestas de sociedades más justas. Involucran también un ejercicio de reflexión y de imaginación acerca del futuro (pues nunca se educa sólo para el presente), y las posibilidades de transformación de ese presente en el que nos inscribimos. Si bien las neurociencias tienen a su favor la evidencia empírica que pueden aportar, podemos afirmar, con respecto a las diferencias cerebrales de niños y niñas, que hasta hoy esa evidencia no es concluyente, ni puede ser utilizada para sugerir con fundamento estrategias neuroeducativas diferenciadas, ni segregación por sexo en el aula. Sólo integrando cuidadosamente los conocimientos de las neurociencias y la educación podemos llegar a conclusiones relevantes acerca de cómo impactan las posibles diferencias cerebrales en las prácticas educativas o, al contrario, cómo las prácticas de enseñanza-aprendizaje pueden influir en tales diferencias (Derks y Krabbendam 2013; Carballo 2016). En espera de que la neuroeducación se convierta en un ámbito de colaboración interdisciplinar bidireccional y con vocación transdisciplinar, ofrecemos esta reflexión y planteamos las cautelas aquí expresadas. ${ }^{8}$

\footnotetext{
${ }^{8}$ Este estudio se inscribe dentro del Proyecto de Investigación Científica y Desarrollo Tecnológico "Neuroeducación moral para las éticas aplicadas" [FFI2016-
} 


\section{BIBLIOGRAFÍA}

Ansari, Daniel, Donna Coch y Bert De Smedt, 2011, "Connecting Education and Cognitive Neuroscience: Where Will the Journey Take Us?", Educational Philosophy and Theory, vol. 43, no. 1, pp. 37-42.

Ansari, Daniel, Bert De Smedt y Ronald Grabner, 2012, "Neuroeducation: A Critical Overview of an Emerging Field", Neuroethics, vol. 5, no. 2, pp. 105-117.

Ayala, Saray, Simone Belli y Fernando Broncano, 2014, "Diferencias, discriminación, cerebro y sexo: controversias científicas de lo social y lo biológico", Encrucijadas, no. 8, pp. 3-9.

Baker, Monya, 2016, "Is There a Reproducibility Crisis?", Nature, no. 533, pp. 452-454.

Balza, Isabel, 2009, "Bioética de los cuerpos sexuados: transexualidad, intersexualidad y transgenerismo", Isegoría, no. 40, pp. 245-258.

Battro, Antonio M. y Daniel P. Cardinali, 1996, Más cerebro en la educación, La Nación, Buenos Aires.

Battro, Antonio M., Kurt Fischer y Pierre Léna (comps.), 2008, The Educated Brain, Cambridge University Press, Cambridge.

Bell, A.D. y S. Variend, 1985, "Failure to Demonstrate Sexual Dimorphism of the Corpus Callosum in Childhood", Journal of Anatomy, no. 143, pp. 143-147.

Bian, Lin, Sarah Leslie y Andrei Cimpian, 2017, "Gender Stereotypes about Intellectual Ability Emerge Early and Influence Children's Interests", Science, no. 27, pp. 389-391.

Bickle, John, 2003, Philosophy and Neuroscience: A Ruthlessly Reductive Account, Kluwer, Dordrecht.

Bing, Janet, 1999, "Brain Sex: How the Media Report and Distort Brain Research", Women and Language, vol. 22, no. 2, pp. 4-12.

Bishop, Katherine y Douglas Wahlsten, 1997, "Sex Differences in the Human Corpus Callosum: Myth or Reality?", Neuroscience and Biobehavioral Reviews, no. 21, pp. 581-601.

Bluhm, Robyn, 2014, "Beyond Neurosexism: Is It Possible to Defend the Female Brain?", en Bluhm, Jacobson y Maibon 2014, pp. 230-245.

Bluhm, Robyn, Anne Jacobson y Heidi Maibon, 2014, Neurofeminism. Issues at the Intersection of Feminist Theory and Cognitive Science, Palgrave Macmillan, Nueva York.

Brizendine, Louan, 2006, The Female Brain, Broadway, Nueva York.

Brożek, Bartosz, 2011, "Philosophy in Neuroscience", en Brożek, Mączka y Grygiel 2011, pp. 163-188.

Brożek, Bartosz, Mączka Janusza y Wojciecha P. Grygiel, 2011, Philosophy in Science: Methods and Applications, Copernicus Press, Cracovia.

76753-C2-2-P], financiado por el Ministerio de Economía, Industria y Competitividad del Gobierno de España. 
Bruer, John, 2008, "Building Bridges in Neuroeducation", en Battro, Fischer y Léna 2008, pp. 43-58.

— 1997, "Education and the Brain: A Bridge Too Far", Educational Researcher, vol. 26, no. 8, pp. 1-13.

Busso, Daniel y Courtney Pollack, 2013, "No Brain Left Behind: Consequences of Neuroscience Discourse for Education", Journal Learning, Media and Technology, vol. 40, no. 2, pp. 168-186.

Butler, Judith, 2004, Undoing Gender, Routledge, Nueva York. , 1990, Gender Trouble: Feminism and the Subversion of Identity, Routledge, Nueva York.

Button, Katherine, John Ioannidis, Claire Mokrysz, Brian Nosek, Jonathan Flint, Emma Robinson y Marcos Munafò, 2013, "Power Failure: Why Small Sample Size Undermines the Reliability of Neuroscience", Nature Reviews Neuroscience, no. 14, pp. 365-376.

Cahill, Larry 2017, “An Issue Whose Time Has Come", Journal of Neuroscience Research, no. 95, pp. 12-13.

Campos, Anna Lucía, 2010, "Neuroeducación: uniendo las neurociencias y la educación en la búsqueda del desarrollo humano", La Educación. Revista Digital, no. 143, pp. 1-14.

Caplan, Paula y Jeremy Caplan, 1994, Thinking Critically about Research on Sex and Gender, Harper Collins, Nueva York.

Carballo, Anna, 2016, "Neuroeducación: de la ciencia al aula", Aula de Infantil, no. 85, pp. 11-14.

Carothers, Bobbi y Harry Reis, 2013, "Men and Women Are From Earth: Examining the Latent Structure of Gender", Journal of Personality and Social Psychology, vol. 104, no. 2, pp. 385-407.

Castelli, Paula, 2018, “¿Qué modelo interdisciplinar requiere la neuroética?", Recerca. Revista de Pensament i Anàlisi, no. 22, pp. 33-49.

Clark, John, 2015, "Philosophy, Neuroscience and Education", Journal Educational Philosophy and Theory, vol. 47, no. 1, pp. 36-46.

Codina, María José, 2014, "Neuroeducación: reflexiones sobre neurociencia, filosofía y educación", Postconvencionales, vol. 6, no. 7, pp. 164-181.

Cortina, Adela, 2011, Neuroética y neuropolítica: sugerencias para la educación moral, Tecnos, Madrid.

Crifaci, Giuilia, Giusseppe Città, Rossella Raso, Manuel Gentile y Mario Allegra, 2015, "Neuroeducation in the Light of Embodied Cognition: An Innovative Perspective", Recent Advances in Educational Technologies. Proceedings of the 2015 International Conference on Education and Modern Educational Technologies (EMET 2015), Isla de Zante, Grecia, 16-20 de julio de 2015, pp. 21-24.

Dalton, Derek y Mark Ortegren, 2011, "Gender Differences in Ethics Research: The Importance of Controlling for the Social Desirability Response Bias", Journal of Business Ethics, vol. 103, no. 1, pp. 7393. 
Davidson, Richard y Sharon Begley, 2012, El lado emocional de tu cerebro, Destino, Madrid.

Della Sala, Sergio y Mike Anderson, 2012, Neuroscience in Education: The Good, the Bad, and the Ugly, Oxford University Press, Oxford.

Derks, Jeffrey y Lydia Krabbendam, 2013, “Is the Brain the Key to a Better Understanding of Gender Differences in the Classroom?", International Journal of Gender, Science and Technology, vol. 5, no. 3, pp. 281-291.

Donaldson, Henry Herbert, 1895, The Growth of the Brain: A Study of the Nervous System in Relation to Education, Skinner Press, Boston.

Dussauge, Isabelle y Anellis Kaiser, 2012, "Neuroscience and Sex/Gender", Neuroethics, vol. 5, no. 3, pp. 211-215.

Eklund, Anders, Thomas Nichols y Hans Knutsson, 2016, “Cluster Failure: Why fMRI Inferences for Spatial Extent Have Inflated False-Positive Rates", PNAS, vol. 113, no. 28, pp. 7900-7905.

Elbert, Thomas, Christo Pantev, Christian Wienbruch, Brigitte Rockstroh y Edward Taub, 1995, "Increased Cortical Representation of the Fingers of the Left Hand in String Players", Science, vol. 270, no. 5234, pp. 305307.

Eliot, Lise, 2013, "Single-Sex Education and the Brain", Sex Roles, vol. 69, nos. 7-8, pp. 363-381.

—_, 2011, "The Trouble with Sex Differences", Neuron, vol. 72, no. 6, pp. 895-898.

Evers, Kathinka, 2015, "Can We Be Epigenetically Proactive?", en Metzinger y Windt 2015, pp. 1-21.

——, 2010, Neuroética. Cuando la materia se despierta, Katz, Madrid.

Fausto-Sterling, Anne, 2015, "How Else Can We Study Sex Differences in Early Infancy?", Developmental Psychobiology, vol. 58, no. 1, pp. 5-16.

- 2000, Sexing the Body. Gender Politics and the Construction of Sexuality, Basic Books, Nueva York.

Ferrari, Michael, 2011, "What Can Neuroscience Bring to Education?", Educational Philosophy and Theory, vol. 43, no. 1, pp. 31-36.

Fine, Cordelia, 2011, “Will Working Mothers' Brains Explode? The Popular New Genre of Neurosexism", Neuroethics, vol. 1, no. 11, pp. 69-72.

Fine, Cordelia, Rebecca Jordan-Young, Anelis Kaiser y Gina Rippon, 2013, "Plasticity, Plasticity, Plasticity. . and the Rigid Problem of Sex", Trends in Cognitive Sciences, vol. 17, no. 11, pp. 550-551.

——, 2010, Delusions of Gender: How Our Minds, Society and Neurosexism Create Difference, Icon Books, Londres.

Flor, Nuria, 2006, "Los cuerpos ficticios de la biomedicina", AIBR. Revista de Antropología Iberoamericana, vol. 1, no. 1, pp. 103-124.

Fraisse, Genevieve, 2016, Los excesos del género, Cátedra, Madrid.

Garcia-Falgueras, Alicia y Dick Swaab, 2008, "A Sex Difference in the Hypothalamic Uncinate Nucleus: Relationship to Gender Identity", Brain, vol. 131, no. 2, pp. 3132-3146. 
Garcia-Falgueras, Alicia, Helena Pinos, Paloma Collado, Eduardo Pasaro, Rosa Fernández, Cynthia L. Jordan, Santiago Segovia y Antonio Guillamón, 2005, "The Role of Androgen Receptor in CNS Masculinization", Brain Research, vol. 1035, no. 1, pp. 13-23.

García-Marzá, Domingo y Ramón A. Feenstra (comps.), 2013, Ética y neurociencias. La aportación a la política, la economía y la educación, Servei de Publicacions de la Universitat Jaume I, Castelló de la Plana.

Gardner, Howard, 2008, "Quandaries for Neuroeducators", Mind, Brain and Education, vol. 2, no. 4, pp. 165-169.

Gazzaniga, Michael, 2012, ¿Quién manda aquî?: el libre albedrío y la ciencia del cerebro, Paidós, Barcelona.

Geake, John, 2008, "Neuromythologies in Education", Educational Research, vol. 50, no. 2, pp. 123-133.

Gonon, François, Erwan Bezard y Thomas Boraud, 2011, "Misrepresentation of Neuroscience Data Might Give Rise to Misleading Conclusions in the Media: The Case of Attention Deficit Hyperactivity Disorder", PLoS One, vol. 6, no. 1, pp. el4618.

Grabowska, Ana, 2017, "Sex on the Brain: Are Gender-Dependent Structural and Functional Differences Associated with Behavior?", Journal of Neuroscience Research, no. 95, pp. 200-212.

Greenfield, Patricia, 2002, "The Mutual Definition of Culture and Biology in Development", en Keller, Poortinga y Schölmerich 2002, pp. 57-66.

Gurian, Michael y Kathie Stevens, 2011, Boys and Girls Learn Differently!: $A$ Guide for Teachers and Parents, Jossey Bass, San Francisco.

Halpern, Diane y Mary LaMay, 2000, "The Smarter Sex: A Critical Review of Sex Differences in Intelligence", Educational Psychology Review, vol. 12, no. 2, pp. 229-246.

Hanggï, Jürgen, Laszlo Fövenyi, Franziskus Liem, Martin Meyer y Lutz Jäncke, 2014, "The Hypothesis of Neuronal Interconnectivity as a Function of Brain Size", Frontiers in Human Neuroscience, no. 8, p. 915.

Haraway, Donna, 1990, Primate Visions: Gender, Race, and Nature in the World of Modern Science, Routledge, Nueva York.

Hardiman, Mariale, Luke Rinne, Emma Gregory y Julia Yarmolinskaya, 2012, "Neuroethics, Neuroeducation, and Classroom Teaching: Where the Brain Sciences Meet Pedagogy", Neuroethics, vol. 5, no. 2, pp. 135143.

Haslam, Nick y Jennifer Whelan, 2008, "Human Natures: Psychological Essentialism in Thinking about Differences Between People", Social and Personality Psychology. Compass, no. 2, pp. 1297-1312.

Haslam, Nick, Louis Rothschild y Donald Ernst, 2000, "Essencialist Beliefs about Social Categories", British Journal of Social Psychology, no. 39, pp. 113-127.

Hoffman, Ginger y Robyn Bluhm, 2016, "Neurosexism and Neurofeminism", Philosophy Compass, vol. 11, no. 11, pp. 716-729. 
Howard-Jones, Paul, 2014, "Neuroscience and Education: Myths and Messages", Nature Reviews Neuroscience, vol. 15, no. 12, pp. 817-824.

- 2012, Education and Neuroscience: Evidence, Theory and Practical Application, Routledge, Londres.

$-\ldots, 2010$, Introducing Neuroeducational Research: Neuroscience, Education and the Brain from Contexts to Practice, Routledge, Londres/Nueva York.

Howard-Jones, Paul y Kate Fenton, 2012, "The Need for Interdisciplinary Dialogue in Developing Ethical Approaches to Neuroeducational Research", Neuroethics, vol. 5, no. 2, pp. 119-134.

Hyde, Janet, 2007, "New Directions in the Study of Gender Similarities and Differences", Current Directions in Psychological Science, vol. 16, no. 5, pp. 259-263.

—_, 2005, "The Gender Similarities Hypothesis", American Psychologist, vol. 60, no. 6, pp. 581-592.

Illes, Judy y Barbara Sahakian, 2011, The Oxford Handbook of Neuroethics, Oxford University Press, Oxford.

Ingalhalikar, Madhura, Alex Smith, Drew Parker, Theodore Satterwaite, Mark Elliott, Kosha Ruparel, Hakon Hakonarson, Raquel Gur y Ragini Verma, 2014, "Sex Differences in the Structural Connectome of the Human Brain", $P N A S$, vol. 111, no. 2, pp. 823-828.

James, William, 1890, The Principles of Psychology, Holt, Nueva York.

Jensen, Eric, 2010, Cerebro y aprendizaje, Narcea, Madrid.

Joel, Daphna, 2016, "Captured in Terminology: Sex, Sex Categories, and Sex Differences", Feminism and Psychology, vol. 26, no. 3, pp. 335345.

Joel, Daphna y Ricardo Tarrasch, 2014, "On the Mis-Presentation and Misinterpretation of Gender-Related Data: the Case of Ingalhalikar's Human Connectome Study", PNAS, vol. 111, no. 6, p. E637.

Joel, Daphna, Zohar Berman, Ido Tavor, Nadav Wexler, Olga Gaber, Yaniv Stein, Nisan Shefi, Jared Pool, Sebastian Urchs, Daniel S. Margulies, Franziskus Liem, Jürgen Hänggi, Lutz Jäncke y Yaniv Assaf, 2015, "Sex Beyond the Genitalia: the Human Brain Mosaic", $P N A S$, vol. 112, no. 50, pp. 15468-15473.

Johnson, Allan, 1997, The Gender Knot. Unraveling our Patriarchal Legacy, Temple University Press, Filadelfia.

Johnson, David, 2011, Brain Culture: Neuroscience and Popular Media, Rutgers University Press, New Brunswick.

Joldersma, Clarence (comp.), 2016, Neuroscience and Education: A Philosophical Appraisal, Routledge, Nueva York.

Jordan-Young, Rebecca, 2011, Brain Storm: The Flaws in the Science of Sex Differences, Harvard University Press, Cambridge, Mass.

Jordan-Young, Rebecca y Raffaella Rumiati, 2011, "Hardwired for Sexism? Approaches to Sex/Gender in Neuroscience", Neuroethics, vol. 5, no. 3, pp. $305-315$. 
Kaiser, Anelis, 2012, "Re-Conceptualizing 'Sex' and 'Gender' in the Human Brain”, Zeitschrift für Psychologie, vol. 220, no. 2, pp. 130-136.

Kaiser, Anelis, Sven Haller, Sigrid Schmitz y Cordula Nitsch, 2009, "On Sex/Gender Related Similarities and Differences in fMRI Language Research", Brain Research Review, vol. 61, no. 2, pp. 49-59.

Keller, Heidi, Ype Poortinga y Axel Schölmerich (comps.), 2002, Between Culture and Biology. Perspectives on Ontogenetic Development, Cambridge University Press, Cambridge.

Kitchen, William H., 2017, Philosophical Reflections on Neuroscience and Education, Bloomsbury, Londres.

Kraus, Cynthia, 2012, "Critical Studies of the Sexed Brain: A Critique of What and for Whom?", Neuroethics, vol. 5, no. 3, pp. 247-259.

Leonard, Christiana M., Stephen Towler, Suzanne Welcome, Laura Halderman, Ron Otto, Marck Eckert y Christine Chiarello, 2008, "Size Matters: Cerebral Volume Influences Sex Differences in Neuroanatomy", Cerebral Cortex, no. 18, pp. 2920-2931.

Liben, Lynn, 2016, “We've Come a Long Way, Baby (But We're Not There Yet: Gender Past, Present, and Future", Child Development, vol. 87, no. 1, pp. 5-28.

—_, 2015, "Probability Values and Human Values in Evaluating SingleSex Education", Sex Roles, no. 72, pp. 401-426.

Lindsey, Linda, 2015, Gender Roles: A Sociological Perspective, Routledge, Londres/Nueva York.

Lipina, Sebastián, 2014, "Consideraciones neuroéticas de la pobreza infantil", en Salles y Evers 2014, pp. 67-101.

Luders, Eileen y Arthur Toga, 2010, "Sex Differences in Brain Anatomy", Progress in Brain Research, no. 186, pp. 3-12.

Maguire, Eleanor, David Gadian, Ingrid Johnsrude, Catriona Good, John Ashburner, Richard Frackowiak y Cristopher Fritz, 2010, "NavigationRelated Structural Change in the Hippocampi of Taxi Drivers", $P N A S$, vol. 97, no. 8, pp. 4398-4403.

Maney, Donna, 2014, “Just Like a Circus: The Public Consumption of Sex Differences", Ethical Issues in Behavioral Neuroscience, no. 19, pp. 279 296.

Marwha, Dhruv, Meha Halari y Lise Eliot, 2017, "Meta-Analysis Reveals a Lack of Sexual Dimorphism in Human Amygdala Volume", Neuroimage, no. 147, pp. 282-294.

Maxwell, Bruce y Eric Racine, 2012, "The Ethics of Neuroeducation: Research, Practice and Policy", Neuroethics, no. 5, pp. 101-103.

McCabe, David y Alan Castel, 2008, "Seeing Is Believing. The Effect of Brain Images on Judgement of Scientific Reasoning", Cognition, no. 107, pp. 343-352.

Medina-Vicent, Maria, 2016, “Neurociencia y teoría política feminista. La inestabilidad sexo-género-sexualidad a través de la obra de Paul 
B. Preciado", Pensamiento. Revista de Investigación e Información Filosófica, vol. 72, no. 273, pp. 981-996.

Medina-Vicent, Maria y Daniel Pallarés-Domínguez, 2017, "Las huellas del neurosexismo en la literatura popular del management dirigida a mujeres", Política y sociedad, vol. 54, no. 3, pp. 683-705.

Metzinger, T. y J.M. Windt, 2015, Open Mind, Mind Group, Fráncfort del Meno.

Mora, Francisco, 2013, Neuroeducación, Alianza, Madrid.

, 2007, Neurocultura. Una cultura basada en el cerebro, Alianza, Madrid.

Morín, Edgar, 1999, Los siete saberes necesarios para la educación del futuro, Unesco, París.

Northoff, Georg, 2004, "What Is Neurophilosophy? A Methodological Account", Journal for General Philosophy of Science, no. 35, pp. 91127.

Nouri, Ali y Mohammad Mehrmohammadi, 2012, "Defining the Boundaries for Neuroeducation as a Field of Study", Educational Research Journal, vol. 27, nos. 1-2, pp. 1-25.

Pallarés-Domínguez, Daniel, 2016, "El potencial ético y educativo de la epigénesis proactiva en Kathinka Evers", Quaderns de Filosofia, vol. 3, no. 2 , pp. $37-57$.

—_ 2015, "Hacia una conceptualización dialógica de la neuroeducación", Participación educativa, vol. 4, no. 7, pp. 133-142.

— , 2013, "Críticas y orientaciones para el estudio de la neuroética", Recerca. Revista de Pensament $i$ Anàlisi, no. 13, pp. 85-102.

Pallarés-Domínguez, Daniel y Andrés Richart, 2018, "Entre la neuroética y la neuroeducación: las fronteras de las neurociencias sociales", Recerca. Revista de Pensament i Anàlisi, no. 22, pp. 7-13.

Pascual-Leone, Álvaro, Amir Amedi, Felipe Fregni y Lofti Merabet, 2005, "The Plastic Human Brain Cortex", Annual Review of Neuroscience, no. 28 , pp. $377-401$.

Pascual-Leone, Álvaro, Catarina Freitas, Lindsay Oberman, Jared Horvath, Mark Halko, Mark Eldaief, Shahid Bashir, Marine Vernet, Mouhshin Shafi, Brandon Westover, Andrew Vahabzadeh y Alexander Rotenberg, 2011, "Characterizing Brain Cortical Plasticity and Network Dynamics Across the Age-Span in Health and Disease with TMS-EEG and TMSfMRI", Brain Topography, no. 24, pp. 302-315.

Pastor, María Carmen y Eva Cifre (comps.), 2017, Emociones y salud. Una mirada con perspectiva de género, Servei de Publicacions de la Universitat Jaume I, Castelló de la Plana.

Pateman, Carole, 1988, The Sexual Contract, Polity Press, Cambridge.

Patten, Kathryn y Stephen Campbell (comps.), 2011, Educational Neuroscience: Initiatives and Emerging Issues, Wiley, Hoboken. 
Pletxer, Belinda, 2015, "From Sex Differences in Neuroscience to a Neuroscience of Sex Differences: New Directions and Perspectives", Frontiers of Neurosciences, no. 9, pp. 6-9.

Ponti, Giovana, Paolo Peretto y Luca Bonfanti, 2008, “Genesis of Neuronal and Glial Progenitors in the Cerebellar Cortex of Peripuberal and Adult Rabbits", PLoS ONE, vol. 3, no. 6, pp. E2366.

Post Halleck, Reuben, 2010, The Education of the Central Nervous System, Nabu Press, Charleston.

Prager, Eric M., 2017, “An Issue Whose Time Has Come: Sex/Gender Influences on Nervous System Function", Journal of Neuroscience Research, vol. 95, no. 7, p. 11.

Preciado, Paul B, 2002, Manifiesto contra-sexual, Opera Prima, Madrid.

Rakic, Pasko, 2002, "Neurogenesis in Adult Primate Neocortex: An Evaluation of the Evidence", Nature Reviews Neuroscience, vol. 3, no. 1, pp. 65-71.

Reverter-Bañón, Sonia, 2017a, "Repensar la identidad sexo/género. El caso de la intersexualidad", en Pastor y Cifre 2017, pp. 99-109.

, 2017b, "El Neurofeminismo frente a la investigación sobre la diferencia sexual", Daimon. Revista Internacional de Filosofía, Suplemento 6 , pp. 95-110.

—_, 2016, "Reflexión crítica frente al neurosexismo", Pensamiento. Revista de Investigación e Información Filosófica, vol. 72, no. 273, pp. 959-979.

— 2010, "La deriva teórica del feminismo", Daimon. Revista Internacional de Filosofía, Suplemento 3, pp. 153-162.

Rippon, Gina, Rebbecca Jordan-Young, Anelis Kaiser y Cordelia Fine, 2014, "Recommendations for Sex/Gender Neuroimaging Research: Key Principles and Implications for Research Design, Analysis, and Interpretation", Frontiers in Human Neuroscience, no. 8, pp. 1-13.

Salles, Arleen, 2013, "On the Normative Implications of Social Neuroscience", Recerca. Revista de pensament $i$ anàlisi, no. 13, pp. 29-42.

Salles, Arleen y Kathinka Evers (comps.), 2014, La vida social del cerebro, Fontamara, México.

Satterwhite, Theodore, Daniel Wolf, David Roalf, Kosha Ruparell, Guray Erus, Simon Vandekar, Efstathios Gennatas, Mark Elliott, Alex Smith, Hanko Hakonarson, Ragini Verma, Christos Davatzikos y Raquel Gur, 2014, "Linked Sex Differences in Cognition and Functional Connectivity in Youth", Cerebral Cortex, no. 25, pp. 2383-2394.

Schmitz, Sigrid y Gina Höppner, 2014, "Neurofeminism and Feminist Neurosciences: A Critical Review of Contemporary Brain Research", Frontiers in Human Neuroscience, vol. 8, article 546, pp. 1-10.

Schmitz, Sigrid, 2012, "The Neurotechnological Cerebral Subject: Persistence of Implicit and Explicit Gender Norms in a Network of Change", Neuroethics, vol. 5, no. 3, pp. 261-274. 
Schrag, Francis, 2011, “Does Neuroscience Matter for Education?”, Educational Theory, no. 61, pp. 221-237.

Schulz, Pablo C. y Issa Katime, 2003, "Los fraudes científicos", Revista Iberoamericana de Polímeros, vol. 4, no. 2, pp. 1-90.

Scott, Joan, 1896, "Gender: A Useful Category of Historical Analysis", The American Historical Review, vol. 91, no. 5, pp. 1053-1075.

Shaywitz, Bennett, Sally Shaywitz, Kenneth Pug, Todd Constable, Pawell Skudlarwski, Robert Fulbright, Richard Bronen, Jack Feltcher, Donald Shankweller, Leonard Katz y John Gore, 1995, "Sex Differences in the Functional Organization of the Brain for Language", Nature, no. 373, pp. 607-609.

Sommer, Metten, André Aleman, Iris Somers, Marco Boks y René Kahn, 2008, "Sex Differences in Handedness, Asymmetry of the Planum Temporale and Functional Language Lateralization", Brain Res, no. 1206, pp. 76-88.

Sousa, David (comp.), 2011, Educational Neuroscience, Corwin, Thousand Oaks.

Swaab, Dick y Alicia Garcia-Falgueras, 2009, "Sexual Differentiation of the Human Brain in Relation to Gender Identity and Sexual Orientation", Functional Neurology, vol. 24, no. 1, pp. 17-28.

Tan, Anh, Wenli Ma, Amit Vira, Dhruv Marwha y Lise Eliot, 2016, "The Human Hippocampus Is Not Sexually-Dimorphic: Meta-Analysis of Structural MRI Volumes", Neuroimage, no. 124(Pt A), pp. 350-366.

Theodoridou, Zoe y Lazaros Triarhou, 2009, "Fin-de-Siecle Advances in Neuroeducation: Henry Herbert Donaldson and Reuben Post Halleck", Mind, Brain and Education, vol. 3, no. 2, pp. 119-129.

Tokuhama-Espinosa, Tracey, 2011, Mind, Brain, and Education Science, W.W. Norton, Nueva York.

Tubert, Silvia (comp.), 2011, Del sexo al género. Los equívocos de un concepto, Cátedra, Madrid.

Van Anders, Sari y Emily Dunn, 2009, “Are Gonadal Steroids Linked with Orgasm Perceptions and Sexual Assertiveness in Women and Men?", Hormones and Behavior, no. 56, pp. 206-213.

Vidal, Catherine, 2011, "The Sexed Brain: Between Science and Ideology", Neuroethics, vol. 5, no. 3, pp. 295-303.

—_, 2005, "Brain, Sex and Ideology", Diogenes, no. 208, pp. 127-133.

Vidal, Fernando, 2008, "Historical Considerations on Brain and Self", en Battro, Fischer y Léna 2008, pp. 20-42.

Vos, Jan de, 2016, The Metamorphoses of the Brain. Neurologisation and its Discontents, Palgrave Macmillan, Nueva York.

Wexler, Bruce, 2011, "Neuroplasticity, Culture, and Society", en Illes y Sahakian 2011, pp. 743-760.

Recibido el 18 de junio de 2017; revisado el 3 de julio de 2018; aceptado el 17 de agosto de 2018. 